\title{
CHARACTERIZATIONS OF IDEAL CLUSTER POINTS
}

\author{
PAOLO LEONETTI AND FABIO MACCHERONI
}

\begin{abstract}
Given an ideal $\mathcal{I}$ on $\omega$, we prove that a sequence in a topological space $X$ is $\mathcal{I}$-convergent if and only if there exists a "big" $\mathcal{I}$-convergent subsequence. Then, we study several properties and show two characterizations of the set of $\mathcal{I}$-cluster points as classical cluster points of a filters on $X$ and as the smallest closed set containing "almost all" the sequence. As a consequence, we obtain that the underlying topology $\tau$ coincides with the topology generated by the pair $(\tau, \mathcal{I})$.
\end{abstract}

\section{INTRODUCTION}

Following the concept of statistical convergence as a generalization of the ordinary convergence, Fridy [15] introduced the statistical limit points and statistical cluster points of a real sequence $\left(x_{n}\right)$ as generalizations of accumulation points.

A real number $\ell$ is said to be a statistical limit point of $\left(x_{n}\right)$ if there exists a subsequence $\left(x_{n_{k}}\right)$ such that

$$
\lim _{k \rightarrow \infty} x_{n_{k}}=\ell
$$

and the set of indices $\left\{n_{k}: k \in \omega\right\}$ has positive upper asymptotic density (see Section 2 for definitions). Also, $\ell$ is called statistical cluster point provided that

$$
\left\{n \in \omega:\left|x_{n}-\ell\right|<\varepsilon\right\}
$$

has positive upper asymptotic density for every $\varepsilon>0$. He proved, among others, that these concepts are not equivalent.

These notions have been studied in a number of recent papers, see e.g. [4, 8, 17, 23, 25, 30, 34]. Extensions of statistical convergence to more general spaces can be found in [1, 10, 27, 28], and to ideal convergence, see e.g. [5, 12, 19, 22].

Given an ideal $\mathcal{I}$ on the positive integers $\omega$, we investigate various properties of $\mathcal{I}$-cluster points and $\mathcal{I}$-limit points of sequences taking values in topological spaces $(X, \tau)$. The main contributions of the article are:

(i) a new characterization of $\mathcal{I}$-convergence: informally, a sequence $\left(x_{n}\right)$ is $\mathcal{I}$-convergent if and only if there exists a "big" $\mathcal{I}$-convergent subsequence (see Theorem 2.4.(iv) and Corollary 2.5);

2010 Mathematics Subject Classification. Primary: 40A35, 54A20. Secondary: 11B05.

Key words and phrases. Ideal cluster point, ideal convergence, G-ideal, filter base, statistical convergence. 
(ii) the topology generated by the pair $(\tau, \mathcal{I})$ corresponds to the underlying topology $\tau$ (see Theorem 3.8);

(iii) a characterization of $\mathcal{I}$-cluster points as classical "cluster points of the filter" generated by the sequence (see Theorem 4.2);

(iv) a characterization of the set of $\mathcal{I}$-cluster points as the smallest closed set containing "almost all" the sequence (see Theorem 4.3).

\section{Preliminaries}

Let Fin be the collection of finite subsets of $\omega$. The upper asymptotic density of a set $S \subseteq \omega$ is defined by

$$
\mathrm{d}^{\star}(S):=\limsup _{n \rightarrow \infty} \frac{|S \cap[1, n]|}{n}
$$

and we denote by $\mathcal{Z}$ the collection of all $S$ such that $\mathrm{d}^{\star}(S)=0$. Hence, a real number $\ell$ is a statistical cluster point of a given real sequence $\left(x_{n}\right)$ if and only if $\left\{n \in \omega:\left|x_{n}-\ell\right|<\varepsilon\right\}$ does not belong to $\mathcal{Z}$ for every $\varepsilon>0$.

An ideal $\mathcal{I}$ on $\omega$ is a family of subsets of positive integers closed under taking finite unions and subsets of its elements. It is also assumed that $\mathcal{I}$ is different from the power set of $\omega$ and contains all the singletons. It is clear that Fin and $\mathcal{Z}$ are ideals. Many other examples can be found, e.g., in [11, Chapter 1] and [21, Section 2]. Intuitively, an ideal represents the collection of subsets of $\omega$ which are "small" in a suitable sense. We denote by $\mathcal{I}^{\star}:=\left\{A \subseteq \omega: A^{c} \in \mathcal{I}\right\}$ the filter dual of $\mathcal{I}$ and by $\mathcal{I}^{+}$the collection of $\mathcal{I}$-positive sets, that is, the collection of all sets which do not belong to $\mathcal{I}$.

Definition 2.1. Given a topological space $X$, a sequence $x=\left(x_{n}\right)$ is said to be $\mathcal{I}$-convergent to $\ell$, shortened with $x_{n} \rightarrow_{\mathcal{I}} \ell$, whenever $\left\{n: x_{n} \in U\right\} \in \mathcal{I}^{\star}$ for all neighborhoods $U$ of $\ell$. Moreover, let $\Gamma_{x}(\mathcal{I})$ denote the set of $\mathcal{I}$-cluster points of $x$, that is, the set of all $\ell \in X$ such that $\left\{n: x_{n} \in U\right\} \in \mathcal{I}^{+}$for all neighborhoods $U$ of $\ell$.

Ordinary convergence corresponds to Fin-convergence (thus, we shorten $x_{n} \rightarrow_{\text {Fin }}$ $\ell$ with $x_{n} \rightarrow \ell$ ) and statistical convergence to $\mathcal{Z}$-convergence. Now, one may worder whether $\mathcal{I}$-convergence corresponds to ordinary convergence with respect to another topology on the same base set. Essentially, it never happens.

Example 2.2. Let us assume that $\mathcal{I} \neq$ Fin and $X$ is a topological space with at least two distinct points such that its topology $\tau$ is not the trivial topology $\tau_{0}$. Hence, there exists a set $I \in \mathcal{I} \backslash$ Fin; in particular, $I$ is infinite. Fix distinct $a, b \in X$ and define the sequence $\left(x_{n}\right)$ by $x_{n}=a$ whenever $n \notin I$ and $x_{n}=b$ otherwise. It follows by construction that $x_{n} \rightarrow_{\mathcal{I}} a$ in $(X, \tau)$. Let us assume, for the sake of contradiction, there exists a topology $\tau^{\prime}$ such that $x_{n} \rightarrow a$ in $\left(X, \tau^{\prime}\right)$. If there is a $\tau^{\prime}$-neighborhood $U$ of $a$ such that $b \notin U$, then $\left\{n: x_{n} \notin U\right\}=I$. This is impossible, since $I$ is not finite. Hence $b \in U$ whenever $a \in U$. By the 
arbitrariness of $a$ and $b$, we conclude that $\tau^{\prime}=\tau_{0}$. The converse is false: given $U \in \tau \backslash \tau_{0}$ and $u \in U$, then the constant sequence $(u)$ is not $\mathcal{I}$-convergent to $\ell$ provided that $\ell \notin U$.

Other notions of convergence have been defined in literature, considering properties of subsequences of $x$ with sufficiently many elements.

Definition 2.3. Given a topological space $X$, a sequence $x=\left(x_{n}\right)$ is said to be $\mathcal{I}^{\star}$-convergent to $\ell$, shortened with $x_{n} \rightarrow_{\mathcal{I}^{\star}} \ell$, whenever there exists a subsequence $\left(x_{n_{k}}\right)$ such that $x_{n_{k}} \rightarrow \ell$ and $\left\{n_{k}: k \in \omega\right\} \in \mathcal{I}^{\star}$. Moreover, let $\Lambda_{x}(\mathcal{I})$ denote the set of $\mathcal{I}$-limit points of $x$, that is, the set of all $\ell \in X$ such that there exists a subsequence $\left(x_{n_{k}}\right)$ for which $x_{n_{k}} \rightarrow \ell$ and $\left\{n_{k}: k \in \omega\right\} \in \mathcal{I}^{+}$.

At this point, recall that an ideal $\mathcal{I}$ is a $P$-ideal if it is $\sigma$-directed modulo finite sets, i.e., for every sequence $\left(A_{n}\right)$ of sets in $\mathcal{I}$ there exists $A \in \mathcal{I}$ such that $A_{n} \backslash A$ is finite for all $n$; equivalent definitions were given, e.g., in [2, Proposition 1].

Moreover, given infinite sets $A, B \subseteq \omega$ such that $A$ has canonical enumeration $\left\{a_{n}: n \in \omega\right\}$, we say that $\mathcal{I}$ a $G$-ideal if

$$
A_{B}:=\left\{a_{b}: b \in B\right\} \in \mathcal{I}^{\star} \quad \text { if and only if } B \in \mathcal{I}^{\star}
$$

provided that $A \in \mathcal{I}^{\star}$. This condition is strictly related to the so-called "property $(\mathrm{G})$ " considered in [3] and to the definition of invariant and thinnable ideals considered in $[23,24]$. Note that the class of G-ideals contains the ideals generated by $\alpha$-densities with $\alpha \geq-1$ (in particular, $\mathcal{I}_{\mathrm{d}}$ and the collection of logarithmic density zero sets), several summable ideals, and the Pólya ideal, i.e.,

$$
\mathcal{I}_{\mathfrak{p}}:=\left\{S \subseteq \omega: \mathfrak{p}^{\star}(S):=\lim _{s \rightarrow 1^{-}} \limsup _{n \rightarrow \infty} \frac{|S \cap[n s, n]|}{(1-s) n}=0\right\}
$$

see [23, Section 2]. Among other things, the upper Pólya density $\mathfrak{p}^{\star}$ has found a number of remarkable applications in analysis and economic theory, see e.g. [35], [26] and [29].

In this regard, we have the following basic result: points (i)-(ii) can be shown by routine arguments, cf. [1, Theorem 3.1] and [10, Section 2] (we omit details); although not explicit in the literature, point (iii) can be considered folklore, see [20, Theorem 3.2] for the case $X$ being a metric space (we include the proof here for the sake of completeness); lastly, point (iv) provides a new characterization of $\mathcal{I}$-convergence (related results can be found in [3, Theorem 3.4] and [23, Theorem $3.4])$.

Theorem 2.4. Let $X$ be a topological space and $\mathcal{I}$ be an ideal. Then:

(i) $\mathcal{I}$-limits and $\mathcal{I}^{\star}$-limits are unique, provided $X$ is Hausdorff;

(ii) $\mathcal{I}^{\star}$-convergence implies $\mathcal{I}$-convergence;

(iii) $\mathcal{I}$-convergence implies $\mathcal{I}^{\star}$-convergence, provided $X$ is first countable and $\mathcal{I}$ is a P-ideal; 
(iv) A sequence $\left(x_{n}\right) \in X^{\omega}$ is $\mathcal{I}$-convergent if and only if there exists an $\mathcal{I}$ convergent subsequence $\left(x_{n_{k}}\right)$ such that $\left\{n_{k}: k \in \omega\right\} \in \mathcal{I}^{\star}$, provided $\mathcal{I}$ is a G-ideal.

Proof. (iii) Let $\left(x_{n}\right)$ be a sequence taking values in $X$ which is $\mathcal{I}$-convergent to some $\ell \in X$. Then, let $\left(U_{j}\right)$ be a countable decreasing local base at $\ell$ and, for each $j$, define $A_{j}:=\left\{n: x_{n} \notin U_{j}\right\}$. Hence, $A_{j} \in \mathcal{I}$ for each $j,\left(A_{j}\right)$ is increasing, and, since $\mathcal{I}$ is a P-ideal, there exists $A \in \mathcal{I}$ such that $A_{j} \backslash A$ is finite for all $j$. Denoting by $\left(n_{k}\right)$ the increasing sequence of integers in $A^{c}$ (which belongs to $\mathcal{I}^{\star}$ ), it follows that $x_{n_{k}} \rightarrow \ell$. Indeed, letting $V$ be a neighborhood of $\ell$ and $j \in \omega$ such that $U_{j} \subseteq V$, then the finiteness of $\left\{k: x_{n_{k}} \notin V\right\}$ follows by the fact that it has the same cardinality of $\left\{n_{k}: x_{n_{k}} \notin V\right\}$ and $\left\{n_{k}: x_{n_{k}} \notin V\right\} \subseteq\left\{n_{k}: x_{n_{k}} \notin U_{j}\right\} \subseteq$ $\left\{n \in A^{c}: x_{n} \notin U_{j}\right\}=A_{j} \backslash A$.

(iv) Let us suppose that $\left(x_{n}\right)$ is $\mathcal{I}$-convergent to $\ell \in X$. Fix also $I \in \mathcal{I}$ and let $\left(n_{k}\right)$ be the increasing enumeration of $I^{c}$. Then, it is claimed that the subsequence $\left(x_{n_{k}}\right)$ is $\mathcal{I}$-convergent to $\ell$. Indeed, for each neighborhood $U$ of $\ell$, we have $\left\{n: x_{n} \notin\right.$ $U\} \in \mathcal{I}$ by hypothesis, hence $\left\{n_{k}: x_{n_{k}} \in U\right\}=\left\{n: x_{n} \in U\right\} \backslash I=\omega \backslash\left(\left\{n: x_{n} \notin\right.\right.$ $U\} \cup I) \in \mathcal{I}^{\star}$. It follows by the fact that $\mathcal{I}$ is a G-ideal that $\left\{k: x_{n_{k}} \in U\right\} \in \mathcal{I}^{\star}$, that is, $x_{n_{k}} \rightarrow_{\mathcal{I}} \ell$. The converse can be shown similarly.

It is well known that $\mathcal{Z}$ is a P-ideal (see e.g. [13, Proposition 3.2]) and, as recalled before, it is also a G-ideal. Hence:

Corollary 2.5. Let $\left(x_{n}\right)$ be a sequence taking values in a topological space $X$. Then the following are equivalent:

(i) $\left(x_{n}\right)$ is statistically convergent;

(ii) There exists a statistically convergent subsequence $\left(x_{n_{k}}\right)$ with $\left\{n_{k}: k \in\right.$ $\omega\} \in \mathcal{Z}^{\star}$.

If, in addition, $X$ is first countable, then they are also equivalent to:

(iii) There exists a convergent subsequence $\left(x_{n_{k}}\right)$ with $\left\{n_{k}: k \in \omega\right\} \in \mathcal{Z}^{\star}$;

It is worth noting that the equivalence between (i) and (iii) can be already found in [10, Theorem 2.2], cf. also [14, Theorem 1] and [30, Theorem 1].

We obtain also an abstract version of [7, Theorem 2.3], see also [5, Proposition 1] and [33, Theorem 1]; the proof goes verbatim, hence we omit it.

Corollary 2.6. Let $\mathcal{I}$ be a P-ideal and $\left(x_{n}\right)$ be a sequence taking values in a metrizable group (with identity 0 ) such that $x_{n} \rightarrow_{\mathcal{I}} \ell$. Then, there exist sequences $\left(y_{n}\right)$ and $\left(z_{n}\right)$ such that: $x_{n}=y_{n}+z_{n}$ for all $n, y_{n} \rightarrow \ell$, and $\left\{n \in \omega: z_{n} \neq 0\right\} \in \mathcal{I}$.

Recall that a real double sequence $x=\left(x_{n, m}: n, m \in \omega\right)$ has Pringsheim limit $\ell$ provided that for every $\varepsilon>0$ there exists $k \in \omega$ such that $\left|x_{n, m}-\ell\right|<\varepsilon$ for all $n, m \geq k$. Identifying ideals on countable sets with ideals on $\omega$ through a fixed bijection, it is easily seen that this is equivalent to $x \rightarrow_{\mathcal{I}_{\mathrm{Pr}}} \ell$, where $\mathcal{I}_{\mathrm{Pr}}$ is the ideal 
defined by

$$
\mathcal{I}_{\mathrm{Pr}}:=\left\{A \subseteq \omega \times \omega: \limsup _{n \rightarrow \infty} \sup \{k:(n, k) \in A\}<\infty\right\} .
$$

Equivalently, $\mathcal{I}_{\operatorname{Pr}}$ is the ideal on $\omega \times \omega$ containing the complements of $[n, \infty) \times$ $[n, \infty)$ for all $n \in \omega$. At this point, for each $n, m \in \omega$, let $\mu_{n, m}$ be the uniform probability measure on $\{1, \ldots, n\} \times\{1, \ldots, m\}$ and define the ideal

$$
\mathcal{Z}_{\mathrm{Pr}}:=\left\{A \subseteq \omega \times \omega: \mu_{n, m}(A) \rightarrow_{\mathcal{I}_{\mathrm{Pr}}} 0\right\} .
$$

Note that $\mathcal{I}_{\operatorname{Pr}} \subseteq \mathcal{Z}_{\operatorname{Pr}}$ and that $\mathcal{Z}_{\operatorname{Pr}}$ is a P-ideal. The notion of convergence of real double sequences $\left(x_{n, m}\right)$ with respect to the ideal $\mathcal{Z}_{\operatorname{Pr}}$ has been recently introduced in [31, 32]; here, it has been simply defined "statistical convergence" of double sequences. Accordingly, it has been shown in [31, Theorem 2] that a real double sequence $\left(x_{n, m}\right)$ is statistically convergent to $\ell$ if and only if there exist real double sequences $\left(y_{n, m}\right)$ and $\left(z_{n, m}\right)$ such that $y_{n, m} \rightarrow_{\mathcal{I}_{\mathrm{Pr}}} \ell$ and $\left\{(n, m): z_{n, m} \neq 0\right\} \in \mathcal{Z}_{\mathrm{Pr}}$. However, this is an immediate consequence of Corollary 2.6.

\section{IdeAl Cluster points}

Given sequences $x$ and $y$ taking values in a topological space $X$, we say that they are $\mathcal{I}$-equivalent, shortened with $x \equiv_{\mathcal{I}} y$, if $\left\{n: x_{n} \neq y_{n}\right\} \in \mathcal{I}$ (it is easy to see that $\equiv_{\mathcal{I}}$ is an equivalence relation). The following lemmas, which collect and extend several results contained in [10, 15, 20], show some standard properties of $\mathcal{I}$-cluster and $\mathcal{I}$-limit points.

Lemma 3.1. Let $x$ and $y$ be sequences taking values in a topological space $X$ and fix ideals $\mathcal{I} \subseteq \mathcal{J}$. Then:

(i) $\Lambda_{x}(\mathcal{J}) \subseteq \Lambda_{x}(\mathcal{I})$ and $\Gamma_{x}(\mathcal{J}) \subseteq \Gamma_{x}(\mathcal{I})$;

(ii) $\Lambda_{x}($ Fin $)=\Gamma_{x}($ Fin $)$, provided $X$ is first countable;

(iii) $\Lambda_{x}(\mathcal{I}) \subseteq \Gamma_{x}(\mathcal{I})$;

(iv) $\Gamma_{x}(\mathcal{I})$ is closed;

(v) $\Lambda_{x}(\mathcal{I})=\Lambda_{y}(\mathcal{I})$ and $\Gamma_{x}(\mathcal{I})=\Gamma_{y}(\mathcal{I})$ provided $x \equiv_{\mathcal{I}} y$;

(vi) $\Gamma_{x}(\mathcal{I}) \cap K \neq \emptyset$, provided $K \subseteq X$ is compact and $\left\{n: x_{n} \in K\right\} \in \mathcal{I}^{+}$;

(vii) $\Lambda_{x}(\mathcal{I})=\Gamma_{x}(\mathcal{I})=\{\ell\}$ provided $x_{n} \rightarrow_{\mathcal{I}^{\star}} \ell$ and $X$ is Hausdorff.

Proof. (i) and (ii) easily follow from the definitions. In addition, (iii) is obvious if $\Lambda_{x}(\mathcal{I})=\emptyset$. Otherwise, fix $\ell \in \Lambda_{x}(\mathcal{I})$ and a neighborhood $U$ of $\ell$. Then, there exists an increasing subsequence $\left(n_{k}\right)$ with $\left\{n_{k}\right\} \in \mathcal{I}^{+}$such that $x_{n_{k}} \rightarrow \ell$, so that $S:=\left\{n_{k}: x_{n_{k}} \notin U\right\}$ is finite. This implies that $\left\{n_{k}\right\} \backslash S \subseteq\left\{n: x_{n} \in U\right\}$. To conclude, it is sufficient to note that $\left\{n_{k}\right\} \backslash S \notin \mathcal{I}$, therefore $\left\{n: x_{n} \in U\right\} \in \mathcal{I}^{+}$.

Similarly, (iv) is clear if $\Gamma_{x}(\mathcal{I})=\emptyset$. In the opposite, let $y$ be an accumulation point of $\Gamma_{x}(\mathcal{I})$ and $U$ a neighborhood of $y$. Then, there exists $z \in \Gamma_{x}(\mathcal{I}) \cap U$. Let $V$ be a neighborhood of $z$ contained in $U$. Considering that $\left\{n: x_{n} \in V\right\} \subseteq\{n$ : $\left.x_{n} \in U\right\}$ and $\left\{n: x_{n} \in V\right\} \in \mathcal{I}^{+}$, we conclude that $y \in \Gamma_{x}(\mathcal{I})$. 
To prove $(\mathrm{v})$, fix $\ell \in \Lambda_{x}(\mathcal{I})$, so that there exists a subsequence $\left(x_{n_{k}}\right)$ such that $\left\{n_{k}\right\} \in \mathcal{I}^{+}$and $x_{n_{k}} \rightarrow \ell$. Since $\left\{n: x_{n} \neq y_{n}\right\} \in \mathcal{I}$ and $\left\{n_{k}: x_{n_{k}} \neq y_{n_{k}}\right\} \subseteq\{n:$ $\left.x_{n} \neq y_{n}\right\}$, then $S:=\left\{n_{k}: x_{n_{k}}=y_{n_{k}}\right\} \in \mathcal{I}^{+}$. Denoting by $\left(s_{n}\right)$ the canonical enumeration of $S$, we obtain $y_{s_{n}} \rightarrow \ell$, hence $\ell \in \Lambda_{y}(\mathcal{I})$. By the arbitrariness of $\ell$, we have $\Lambda_{x}(\mathcal{I}) \subseteq \Lambda_{y}(\mathcal{I})$ therefore, by symmetry, $\Lambda_{x}(\mathcal{I})=\Lambda_{y}(\mathcal{I})$. The other claim can be shown similarly.

The proof of (vi) can be found in [9, Theorem 6], cf. also [10, Theorem 2.14] for the case $\mathcal{I}=\mathcal{Z}$.

Lastly, suppose that $x_{n} \rightarrow_{\mathcal{I}^{\star}} \ell$ so that $x_{n} \rightarrow_{\mathcal{I}} \ell$ by Theorem 2.4.(ii) and, in particular, $\ell \in \Lambda_{x}(\mathcal{I})$. Also, thanks to (iii), we have $\{\ell\} \subseteq \Lambda_{x}(\mathcal{I}) \subseteq \Gamma_{x}(\mathcal{I})$. To conclude, let us suppose for the sake of contradition that there exists an $\mathcal{I}$-cluster point $\ell^{\prime}$ of $x$ different from $\ell$. Fix disjoint neighborhoods $U$ and $U^{\prime}$ of $\ell$ and $\ell^{\prime}$, respectively. On the one hand, since $\ell^{\prime}$ is a $\mathcal{I}$-cluster point, then $\left\{n: x_{n} \in U^{\prime}\right\} \in$ $\mathcal{I}^{+}$. On the other hand, this is impossible since $\left\{n: x_{n} \in U^{\prime}\right\} \subseteq\left\{n: x_{n} \notin U\right\} \in \mathcal{I}$. This proves (vii).

It follows at once from Theorem 2.4.(iii) and Lemma 3.1.(vii) that:

Corollary 3.2. Let $\mathcal{I}$ be a P-ideal and $\left(x_{n}\right)$ be a sequence taking values in a first countable Hausdorff space such that $x_{n} \rightarrow_{\mathcal{I}} \ell$. Then $\Lambda_{x}(\mathcal{I})=\Gamma_{x}(\mathcal{I})=\{\ell\}$.

The converse of Corollary 3.2 does not hold in general: the real sequence $x$ defined by $x_{n}=n$ if $n$ is even and $x_{n}=0$ otherwise satisfies $\Lambda_{x}(\mathcal{Z})=\Gamma_{x}(\mathcal{Z})=\{0\}$ while $x_{n} \dashv_{\mathcal{Z}} 0$. On the other hand, if the underlying space space is compact, it is sufficient, cf. [16, Proposition 8] for a special case.

Lemma 3.3. Let $\mathcal{I}$ be an ideal, let $\left(x_{n}\right)$ be a sequence in a first countable compact space $X$, and suppose that $\Gamma_{x}(\mathcal{I})=\{\ell\}$. Then $x_{n} \rightarrow_{\mathcal{I}} \ell$. In addition, if $\mathcal{I}$ is a $P$-ideal, then $x_{n} \rightarrow_{\mathcal{I}^{\star}} \ell$.

Proof. Let $\left(U_{k}\right)$ be a decreasing local base at $\ell$. Fix $k \in \omega$ and, for each $z \in X$ with $z \neq \ell$, there exists a neighborhood $U_{z}$ of $z$ such that $\left\{n \in \omega: x_{n} \in U_{z}\right\} \in \mathcal{I}$. Since $\left\{U_{z}: z \in X \backslash\{\ell\}\right\} \cup U_{k}$ is an open cover of $X$ and $X$ is compact, there exists a finite subcover $U_{z_{1}} \cup \cdots \cup U_{z_{m}} \cup U_{k}$; note that $U_{k}$ belongs to the subcover, indeed, in the opposite, we would have $\omega=\bigcup_{i \leq m}\left\{n: x_{n} \in U_{z_{i}}\right\} \in \mathcal{I}$. In particular, $\left\{n \in \omega: x_{n} \in U_{k}\right\} \in \mathcal{I}^{\star}$. Therefore $x_{n} \rightarrow_{\mathcal{I}} \ell$.

If, in addition, $\mathcal{I}$ is a P-ideal then $A_{k}:=\left\{n \in \omega: x_{n} \notin U_{k}\right\}$ is an increasing sequence in $\mathcal{I}$, hence there exists $A \in \mathcal{I}$ such that $A_{k} \backslash A \in$ Fin for all $k$. It follows that $\left\{n \in A^{c}: x_{n} \notin U_{k}\right\}=A_{k} \cap A^{c} \in$ Fin for all $k$, that is, $x_{n} \rightarrow_{\mathcal{I}^{\star}} \ell$.

As an application, we obtain a generalization of [17, Theorem 3]:

Corollary 3.4. Let $\mathcal{I}$ be an ideal and $\left(x_{n}\right)$ be a sequence in first countable space $X$ such that $\left\{n \in \omega: x_{n} \notin K\right\} \in \mathcal{I}$ for some compact $K \subseteq X$. Then $x_{n} \rightarrow_{\mathcal{I}} \ell$ if and only if $\Gamma_{x}(\mathcal{I})=\{\ell\}$.

Moreover, Lemma 3.1.(v) can be strenghtened if $X$ is a topological group: 
Lemma 3.5. Let $x$ and $y$ be sequences taking values in a topological group $X$ (written additively, with identity 0 ) and fix an ideal $\mathcal{I}$. Then:

(i) $\Gamma_{x}(\mathcal{I})=\Gamma_{y}(\mathcal{I})$ provided $x_{n}-y_{n} \rightarrow_{\mathcal{I}} 0$;

(ii) $\Lambda_{x}(\mathcal{I})=\Lambda_{y}(\mathcal{I})$ provided $x_{n}-y_{n} \rightarrow_{\mathcal{I}^{\star}} 0$.

Proof. Let $z$ be the sequence defined by $z_{n}=x_{n}-y_{n}$.

(i) It follows by hypothesis $z_{n} \rightarrow_{\mathcal{I}} 0$ and $-z_{n} \rightarrow_{\mathcal{I}} 0$. Fix $\ell \in \Gamma_{x}(\mathcal{I})$ and let $U$ be a neighborhood of $\ell$. By the continuity of the operation of the group, there exist neighborhoods $V$ and $W$ of $\ell$ and 0 , respectively, such that $V+W \subseteq U$. Considering that $\left\{n: x_{n} \in V\right\} \in \mathcal{I}^{+}$and $\left\{n:-z_{n} \in W\right\} \in \mathcal{I}^{\star}$, it follows that

$$
\left\{n: y_{n} \in U\right\}=\left\{n: x_{n}-z_{n} \in U\right\} \supseteq\left\{n: x_{n} \in V\right\} \cap\left\{n:-z_{n} \in W\right\} \in \mathcal{I}^{+} .
$$

Since $\ell$ and $U$ were arbitrarily chosen, then $\Gamma_{x}(\mathcal{I}) \subseteq \Gamma_{y}(\mathcal{I})$. The opposite inclusion can be shown similarly.

(ii) By hypothesis $z_{n} \rightarrow_{\mathcal{I}^{\star}} 0$ and $-z_{n} \rightarrow_{\mathcal{I}^{\star}} 0$. Fix $\ell \in \Lambda_{x}(\mathcal{I})$, hence there exist $A, B \in \mathcal{I}^{\star}$ such that $\lim _{a \in A} x_{a}=\ell$ and $\lim _{b \in B}-z_{b}=0$. Setting $C:=A \cap B \in \mathcal{I}^{\star}$, it follows that $\lim _{c \in C} y_{c}=\lim _{c \in C} x_{c}-z_{c}=\ell$, therefore $\Lambda_{x}(\mathcal{I}) \subseteq \Lambda_{y}(\mathcal{I})$. The opposite inclusion can be shown similarly.

We recall that, under suitable assumptions on $X$ and $\mathcal{I}$, the collection of $\mathcal{I}$ cluster and $\mathcal{I}$-limit point sets can be characterized as the closed sets and $F_{\sigma}$ sets, respectively; see [4, Theorem 3.1], [10, Section 2], [19, Theorem 1.1], and [20, Section 4]. Moreover, the continuity of the map $x \mapsto \Gamma_{x}(\mathcal{I})$ has been investigated in [19].

The next result establishes a connection between sets of cluster points with respect to different ideals (the proof is based on [15, Theorem 2] which focuses on the case $X=\mathbf{R}, \mathcal{I}=\mathcal{Z}$, and $\mathcal{J}=$ Fin).

Lemma 3.6. Let $x$ be a sequence taking values in a strongly Lindelöf space $X$ and fix ideals $\mathcal{J} \subseteq \mathcal{I}$ such that $\mathcal{I}$ is a P-ideal. Then, there exists an $\mathcal{I}$-equivalent sequence $y$ such that $\Gamma_{x}(\mathcal{I})=\Gamma_{y}(\mathcal{J})$ and $\left\{y_{n}: n \in \omega\right\} \subseteq\left\{x_{n}: n \in \omega\right\}$.

Proof. The claim is obvious if $\Gamma_{x}(\mathcal{I})=\Gamma_{x}(\mathcal{J})$. Hence, let us suppose that $\Delta:=$ $\Gamma_{x}(\mathcal{J}) \backslash \Gamma_{x}(\mathcal{I}) \neq \emptyset$ and, for each $z \in \Delta$, let $U_{z}$ be a neighborhood of $z$ such that $\left\{n: x_{n} \in U_{z}\right\} \in \mathcal{I}$. Then $\bigcup U_{z}$ is an open cover of $\Delta$. Since $X$ is strongly Lindelöf, there exists a countable subset $\left\{z_{k}: k \in \omega\right\} \subseteq \Delta$ such that $\bigcup U_{z_{k}}$ is an open subcover of $\Delta$. Moreover, since $\mathcal{I}$ is a P-ideal, there exists $I \in \mathcal{I}$ such that $\left\{n: x_{n} \in U_{z_{k}}\right\} \backslash I$ is finite for all $k$. At this point, let $\left(i_{n}\right)$ be the canonical enumeration of $\omega \backslash I$ and define the sequence $y$ by $y_{n}=x_{i_{n}}$ if $n \in I$ and $y_{n}=x_{n}$ otherwise. Since $\left\{n: x_{n} \neq y_{n}\right\} \subseteq I \in \mathcal{I}$, then $x \equiv_{\mathcal{I}} y$, hence we obtain by Lemma 3.1.(v) that $\Gamma_{x}(\mathcal{I})=\Gamma_{y}(\mathcal{I})$. The claim follows by the fact that every $\mathcal{J}$-cluster point of $y$ is also an $\mathcal{I}$-cluster point, therefore $\Gamma_{y}(\mathcal{I})=\Gamma_{y}(\mathcal{J})$.

Lastly, given a topological space $(X, \tau)$ and an ideal $\mathcal{I}$, define the family

$$
\tau(\mathcal{I}):=\left\{F^{c} \subseteq X: F=\bigcup_{x \in F^{\omega}} \Gamma_{x}(\mathcal{I})\right\},
$$


that is, $F$ is $\tau(\mathcal{I})$-closed if and only if it is the union of $\mathcal{I}$-cluster points of $F$-valued sequences. In particular, it is immediate that $\tau=\tau($ Fin $)$.

Lemma 3.7. $\tau \subseteq \tau(\mathcal{I})$.

Proof. Let $F$ be a $\tau$-closed set. Thanks to Lemma 3.1.(i), we have

$$
F \subseteq \bigcup_{x \in F^{\omega}} \Gamma_{x}(\mathcal{I}) \subseteq \bigcup_{x \in F^{\omega}} \Gamma_{x}(\text { Fin })=F,
$$

where the first inclusion is obtained by choosing the constant sequence $(f)$, for each fixed $f \in F$. Therefore, $F^{c} \in \tau(\mathcal{I})$.

The converse holds under some additional assumptions:

Theorem 3.8. Assume that one of the following conditions holds:

(i) $X$ is sequentially strongly Lindelöf and $\mathcal{I}$ is a P-ideal;

(ii) $X$ is first countable.

Then $\tau=\tau(\mathcal{I})$.

Proof. Thanks to Lemma 3.7, it is sufficient to show that $\tau(\mathcal{I}) \subseteq \tau$. Let $F$ be a $\tau(\mathcal{I})$-closed set. Then, it is enough to show that if $\ell \in F$ is an $\mathcal{I}$-cluster point of some $F$-valued sequence $x$, it is also an ordinary limit point of some $F$-valued sequence $y$.

(i) This follows directly by Lemma 3.6, setting $\mathcal{J}=$ Fin.

(ii) Let $\left(U_{k}\right)$ be a decreasing local base at $\ell$. Then, there exists a subsequence $\left(x_{n_{k}}\right)$ converging to $\ell$ : to this aim, set $S_{k}:=\left\{n: x_{n} \in U_{k}\right\}$ for each $k$, fix $n_{1} \in S_{1}$ arbitrarily and, for each $k \in \omega$, define $n_{k+1}:=\min S_{k+1} \backslash\left\{1, \ldots, n_{k}\right\}$ (note that this is possible since each $S_{k}$ is infinite).

\section{Characterizations}

Given an ideal $\mathcal{I}$ and a sequence $x$ taking values in a topological space $X$, we define the $\mathcal{I}$-filter generated by $x$ as

$$
\mathscr{F}_{x}(\mathcal{I}):=\left\{Y \subseteq X:\left\{n: x_{n} \notin Y\right\} \in \mathcal{I}\right\} .
$$

It is immediate that $\mathscr{F}_{x}(\mathcal{I})$ is a filter on $X$ with filter base

$$
\mathcal{B}_{x}(\mathcal{I}):=\left\{\left\{x_{n}: n \notin I\right\}: I \in \mathcal{I}\right\} .
$$

In addition, if $\mathcal{I}=$ Fin, then $\mathscr{F}_{x}(\mathcal{I})$ coincides with the standard filter generated by $x$, cf. [6, Definition 7, p.64].

With this notation, we are going to show that $\ell$ is an $\mathcal{I}$-cluster point of $x$ if and only if it is a cluster point of the filter $\mathscr{F}_{x}(\mathcal{I})$, that is, $\ell$ lies in the closure of all sets in the filter base $\mathcal{B}_{x}(\mathcal{I})$, c.f. [6, Definition 2, p.69].

Lemma 4.1. $\bigcap_{B \in \mathcal{B}_{x}(\mathcal{I})} \bar{B} \subseteq \Gamma_{x}(\mathcal{I})$. 
Proof. Let us suppose that $\ell \in \bigcap_{I \in \mathcal{I}} \overline{\left\{x_{n}: n \notin I\right\}}$, that is, for each $I \in \mathcal{I}$ there exists a subsequence $\left(x_{n_{k}}\right)$ converging to $\ell$ such that $\left\{n_{k}: k \in \omega\right\} \cap I=\emptyset$. Suppose for the sake of contradiction that $\ell$ is not an $\mathcal{I}$-cluster point, i.e., there exists an open neighborhood $U$ of $\ell$ such that $J:=\left\{n: x_{n} \in U\right\}$ belongs to $\mathcal{I}$. Then, it follows that $\left\{x_{n}: n \notin J\right\} \in \mathcal{B}_{x}(\mathcal{I})$ hence

$$
\ell \in \bigcap_{B \in \mathcal{B}_{x}(\mathcal{I})} \bar{B} \subseteq \overline{\left\{x_{n}: n \notin J\right\}}=\overline{\left\{x_{n}: x_{n} \notin U\right\}} \subseteq U^{c},
$$

which is impossible since $\ell \in U$.

However, if $X$ is first countable, then also the converse holds.

Theorem 4.2. Let $\mathcal{I}$ be an ideal and $x$ be a sequence taking values in a first countable space $X$. Then $\Gamma_{x}(\mathcal{I})=\bigcap_{B \in \mathcal{B}_{x}(\mathcal{I})} \bar{B}$.

Proof. Thanks to Lemma 4.1, it is sufficient to show that $\Gamma_{x}(\mathcal{I}) \subseteq \bigcap_{B \in \mathcal{B}_{x}(\mathcal{I})} \bar{B}$. Let us suppose that $\ell$ is an $\mathcal{I}$-cluster point of $x$ and fix a decreasing local base $\left(U_{k}\right)$ at $\ell$, so that $S_{k}:=\left\{n: x_{n} \in U_{k}\right\} \in \mathcal{I}^{+}$for all $k$. Fix also $I \in \mathcal{I}$ and note that $T_{k}:=S_{k} \backslash I \in \mathcal{I}^{+}$for all $k$ (in particular, each $T_{k}$ is infinite). Then, we have to prove that $\ell \in \overline{\left\{x_{n}: n \notin I\right\}}$, i.e., there exists a subsequence $\left(x_{n_{k}}\right)$ converging to $\ell$ such that $n_{k} \notin I$ for all $k$. To this aim, it is enough to fix $n_{1} \in T_{1}$ arbitrarily and $n_{k+1}:=\min T_{k+1} \backslash\left\{1, \ldots, n_{k}\right\}$ for all $k \in \omega$. It follows by construction that $\lim _{k \rightarrow \infty} x_{n_{k}}=\ell$ and $n_{k} \notin I$ for all $k$.

As a corollary, we obtain another proof of Lemma 3.1.(iv), provided $X$ is first countable.

We conclude with another characterization of the set of $\mathcal{I}$-cluster points, which subsumes the results contained in [18].

Theorem 4.3. Let $x$ be a sequence taking values in a regular Hausdorff space $X$ such that $\left\{n: x_{n} \notin K\right\} \in \mathcal{I}$ for some compact set $K$. Then $\Gamma_{x}(\mathcal{I})$ is the smallest closed set $C$ such that $\left\{n: x_{n} \notin U\right\} \in \mathcal{I}$ for all sets $U$ containing $C$.

Proof. Fix $\kappa \in K$ and define the sequence $y$ by $y_{n}=\kappa$ if $x_{n} \notin K$ and $y_{n}=x_{n}$ otherwise. It follows by Lemma 3.1.(vi)-(v) that $\emptyset \neq \Gamma_{x}(\mathcal{I})=\Gamma_{y}(\mathcal{I}) \subseteq K$. Let also $\mathscr{C}$ be the family of closed sets $C$ such that $\left\{n: x_{n} \notin U\right\} \in \mathcal{I}$ for all open subsets $U \supseteq C$ (note that $\left\{n: x_{n} \notin U\right\} \in \mathcal{I}$ if and only if $\left\{n: y_{n} \notin U\right\} \in \mathcal{I}$ ).

First, we show that $\Gamma_{x}(\mathcal{I}) \in \mathscr{C}$. Indeed, $\Gamma_{x}(\mathcal{I})$ is closed by Lemma 3.1.(iv); moreover, let us suppose for the sake of contradiction that there exists an open set $U$ containing $\Gamma_{x}(\mathcal{I})$ such that $\left\{n: x_{n} \notin U\right\} \in \mathcal{I}^{+}$, that is, $\left\{n: y_{n} \notin U\right\}=$ $\left\{n: y_{n} \in K \backslash U\right\} \in \mathcal{I}^{+}$. Considering that $K \backslash U$ is compact, we obtain by Lemma 3.1.(vi) that there exists an $\mathcal{I}$-cluster point of $y$ in $K \backslash U$. This contradicts the fact that $\Gamma_{y}(\mathcal{I})=\Gamma_{x}(\mathcal{I}) \subseteq U$.

Lastly, fix $C \in \mathscr{C}$ and let us suppose that $\Gamma_{x}(\mathcal{I}) \backslash C \neq \emptyset$. Fix $\ell \in \Gamma_{x}(\mathcal{I}) \backslash C$ and, by the regularity of $X$, there exist disjoint open sets $U$ and $V$ containing the closed sets $\{\ell\}$ and $K \cap C$, respectively. This is impossible, indeed the set 
$\left\{n: x_{n} \in V\right\}$ belongs to $\mathcal{I}$ since $C \in \mathscr{C}$, and, on the other hand, it contains $\left\{n: x_{n} \in U\right\} \in \mathcal{I}^{+}$since $\ell$ is an $\mathcal{I}$-cluster point.

Acknowledgments. The authors are grateful to Szymon Głab (Łódź University of Technology, PL) and Ondřej Kalenda (Charles University, Prague) for several useful comments.

\section{REFERENCES}

1. H. Albayrak and S. Pehlivan, Statistical convergence and statistical continuity on locally solid Riesz spaces, Topology Appl. 159 (2012), no. 7, 1887-1893.

2. M. Balcerzak, K. Dems, and A. Komisarski, Statistical convergence and ideal convergence for sequences of functions, J. Math. Anal. Appl. 328 (2007), no. 1, 715-729.

3. M. Balcerzak, Sz. Głab, and A. Wachowicz, Qualitative properties of ideal convergent subsequences and rearrangements, Acta Math. Hungar. 150 (2016), no. 2, 312-323.

4. M. Balcerzak and P. Leonetti, On the relationship between ideal cluster points and ideal limit points, Topology Appl. 252 (2019), 178-190.

5. P. Barbarski, R. Filipów, N. Mrożek, and P. Szuca, Uniform density u and $\mathscr{I}_{u}$-convergence on a big set, Math. Commun. 16 (2011), no. 1, 125-130.

6. N. Bourbaki, General topology. Chapters 1-4, Elements of Mathematics (Berlin), SpringerVerlag, Berlin, 1998, Translated from the French, Reprint of the 1989 English translation.

7. J. Connor, The statistical and strong p-Cesàro convergence of sequences, Analysis 8 (1988), no. 1-2, 47-63.

8. J. Connor and J. Kline, On statistical limit points and the consistency of statistical convergence, J. Math. Anal. Appl. 197 (1996), no. 2, 392-399.

9. P. Das, Some further results on ideal convergence in topological spaces, Topology Appl. 159 (2012), no. 10-11, 2621-2626.

10. G. Di Maio and L. D. R. Kočinac, Statistical convergence in topology, Topology Appl. 156 (2008), no. 1, 28-45.

11. I. Farah, Analytic quotients: theory of liftings for quotients over analytic ideals on the integers, Mem. Amer. Math. Soc. 148 (2000), no. 702, xvi+177.

12. R. Filipów, N. Mrożek, I. Recław, and P. Szuca, Ideal convergence of bounded sequences, J. Symbolic Logic 72 (2007), no. 2, 501-512.

13. A. R. Freedman and J. J. Sember, Densities and summability, Pacific J. Math. 95 (1981), no. $2,293-305$.

14. J. A. Fridy, On statistical convergence, Analysis 5 (1985), no. 4, 301-313.

15. _ Statistical limit points, Proc. Amer. Math. Soc. 118 (1993), no. 4, 1187-1192.

16. J. A. Fridy and J. Li, Matrix transformations of statistical cores of complex sequences, Analysis (Munich) 20 (2000), no. 1, 15-34.

17. J. A. Fridy and C. Orhan, Statistical limit superior and limit inferior, Proc. Amer. Math. Soc. 125 (1997), no. 12, 3625-3631.

18. A. Güncan, M. A. Mamedov, and S. Pehlivan, Statistical cluster points of sequences in finite dimensional spaces, Czechoslovak Math. J. 54(129) (2004), no. 1, 95-102.

19. P. Kostyrko, M. Mačaj, T. Šalát, and O. Strauch, On statistical limit points, Proc. Amer. Math. Soc. 129 (2001), no. 9, 2647-2654.

20. P. Kostyrko, T. Šalát, and W. Wilczyński, I-convergence, Real Anal. Exchange 26 (2000/01), no. 2, 669-685.

21. A. Kwela and J. Tryba, Homogeneous ideals on countable sets, Acta Math. Hungar. 151 (2017), no. 1, 139-161. 
22. P. Leonetti, Continuous projections onto ideal convergent sequences, Results in Math., to appear.

23. ㄴ Thinnable ideals and invariance of cluster points, Rocky Mountain J. Math. 48 (2018), no. 6, 1951-1961.

24. _ Invariance of ideal limit points, Topology Appl. 252 (2019), 169-177.

25. P. Leonetti, H. Miller, and Miller van Wieren L., Duality between measure and category of almost all subsequences of a given sequence, Period. Math. Hungar., to appear.

26. N. Levinson, Gap and Density Theorems, American Mathematical Society Colloquium Publications, v. 26, American Mathematical Society, New York, 1940.

27. I. J. Maddox, Statistical convergence in a locally convex space, Math. Proc. Cambridge Philos. Soc. 104 (1988), no. 1, 141-145.

28. M. A. Mamedov and S. Pehlivan, Statistical cluster points and turnpike theorem in nonconvex problems, J. Math. Anal. Appl. 256 (2001), no. 2, 686-693.

29. M. Marinacci, An axiomatic approach to complete patience and time invariance, J. Econom. Theory 83 (1998), no. 1, 105-144.

30. H. I. Miller, A measure theoretical subsequence characterization of statistical convergence, Trans. Amer. Math. Soc. 347 (1995), no. 5, 1811-1819.

31. F. Móricz, Statistical convergence of multiple sequences, Arch. Math. (Basel) 81 (2003), no. $1,82-89$.

32. M. Mursaleen and O. H. H. Edely, Statistical convergence of double sequences, J. Math. Anal. Appl. 288 (2003), no. 1, 223-231.

33. A. Nabiev, S. Pehlivan, and M. Gürdal, On I-Cauchy sequences, Taiwanese J. Math. 11 (2007), no. 2, 569-576.

34. F. Nuray and W. H. Ruckle, Generalized statistical convergence and convergence free spaces, J. Math. Anal. Appl. 245 (2000), no. 2, 513-527.

35. G. Pólya, Untersuchungen über Lücken und Singularitäten von Potenzreihen, Math. Z. 29 (1929), no. 1, 549-640.

Università "Luigi Bocconi", Department of Decision Sciences, Milan, Italy

E-mail address: paolo.leonetti@unibocconi.it

Università "Luigi Bocconi", Department of Decision Sciences, Milan, Italy

E-mail address: fabio.maccheroni@unibocconi.it 